\title{
MiDAS ENCORE: Randomized Controlled Study Design and Protocol
}

\author{
Ramsin M. Benyamin, MD'1, and Peter S. Staats, MD²
}

From: ${ }^{1}$ Millennium Pain Center, Bloomington, IL; ${ }^{2}$ Premier Pain Centers, Shrewsbury, NJ.

Address Correspondence: Ramsin M. Benyamin, MD,

President

Millennium Pain Center

1015 South Mercer Ave.

Bloomington, IL 61701

E-mail:

RBenyamin@

millenniumpaincenter.com

Disclaimer: Sponsor paid independent contractor.

Conflict of interest: Each

author certifies that he or

she, or a member of his or

her immediate family, has no

commercial association (i.e., consultancies, stock ownership, equity interest, patent/licensing arrangements, etc.) that might

pose a conflict of interest in connection with the submitted manuscript.

Manuscript received: o6-10-2015

Accepted for publication: o6-17-2015

Free full manuscript: www.painphysicianjournal.com
Background: Epidural steroid injections (ESIs) are commonly used for treatment of symptomatic lumbar spinal stenosis (LSS). ESIs are generally administered after failure of conservative therapy. For LSS patients suffering from neurogenic claudication, the mild ${ }^{\circledR}$ procedure provides an alternative to ESIs via minimally invasive lumbar decompression. Both ESIs and mild offer interventional pain treatment options for LSS patients experiencing neurogenic claudication refractory to more conservative therapies.

Study Design: Prospective, multi-center, randomized controlled, clinical study.

Setting: Twenty-six interventional pain management centers throughout the United States.

Objective: To compare patient outcomes following treatment with either mild or ESIs in LSS patients with neurogenic claudication and having verified ligamentum flavum hypertrophy.

Methods: Study participants include Medicare beneficiaries who meet study inclusion/exclusion criteria. Eligible patients will be randomized in a 1:1 ratio to one of 2 treatment arms, mild (treatment group) or ESI (control group). Each study group will include approximately 150 patients who have experienced neurogenic claudication symptoms for $\geq 3$ months duration who have failed to respond to physical therapy, home exercise programs, and oral analgesics. Those randomized to mild are prohibited from receiving lumbar ESIs during the study period, while those randomized to ESI may receive ESIs up to 4 times per year. Patient assessments will occur at baseline, 6 months, and one year. An additional assessment will be conducted for the mild patient group at 2 years.

Outcome Measures: The primary efficacy outcome measure is the proportion of Oswestry Disability Index $(\mathrm{ODI})$ responders from baseline to one year follow-up in the treatment group (mild) versus the control group (ESI). ODI responders are defined as those patients achieving the validated Minimal Important Change (MIC) of $\geq 10$ point improvement in ODI from baseline to follow-up as a clinically significant efficacy threshold. Secondary efficacy outcome measures include the proportion of Zurich Claudication Questionnaire (ZCQ) and Numeric Pain Rating Scale (NPRS) responders from baseline to follow-up using validated MIC thresholds. Improvement in ZCQ domains of $\geq 0.5$ is considered significant, and a Patient Satisfaction score of at least 2.5 represents a satisfied patient. A reduction of $\geq 2$ points in NPRS is considered significant pain relief. The primary safety outcome measure is the incidence of device- and/or procedure-related adverse events.

Results: Descriptive summaries will be presented by randomized group for all outcome measures at baseline and follow-up time points. Inferential statistical analysis will be conducted to determine significant differences related to functional improvement, pain relief, and safety outcomes. Primary study results will be presented based on one-year follow-up data, with an interim analysis report when 6-month follow-up data become available.

Limitations: Patients are not blinded due to significant differences in treatment protocols between study groups. Also, since neither study arm is focused on treatment of radicular pain, there may be a higher non-responder rate for both groups versus standard of care due to study restrictions on adjunctive pain therapies.

Conclusions: This prospective, multi-center, randomized controlled study will provide Level I evidence of the safety and effectiveness of mild versus ESIs in managing neurogenic claudication symptoms in LSS patients.

Key words: mild, lumbar spinal stenosis, LSS, epidural steroid injection, neurogenic claudication, ligamentum flavum

Pain Physician 2015; 18:307-316 
umbar spinal stenosis (LSS) is a serious chronic and progressive degenerative condition of the spine which is associated with significant pain and disability. There are many types of central stenosis, but it generally occurs from a combination of degenerative changes occurring in the lumbar spine including hypertrophy of the ligamentum flavum, facet joint arthritic changes, and intervertebral disc bulges. These degenerative changes constrict the space within the spinal canal, causing compression of the lumbar neural elements. This compression often leads to symptomatic neurogenic claudication defined as considerable pain and discomfort in the lower back and legs which is worsened by standing or walking, and relieved by lumbar flexion or sitting $(1,2)$.

Conservative management often includes physical therapy and oral analgesics, and may also involve chiropractic manipulation, acupuncture, or a range of other treatment options focused on pain relief. In the event conservative therapies do not provide adequate relief of neurogenic claudication symptoms, epidural steroid injections (ESIs) are commonly administered as the next step for these patients (3-11). The clinical goal of the mild procedure is to improve pain and function for a particular type of LSS patient suffering from neurogenic claudication-those with hypertrophic ligamentum flavum as a contributing factor. The mild procedure provides minimally invasive lumbar decompression via debulking of the lamina and ligamentum flavum (Vertos Medical, Inc., Aliso Viejo, CA). As interventional pain procedures, mild and ESIs provide therapy options for patients seeking more robust treatment than is available through traditional conservative management. Clinical experience with the mild procedure has been previously reported (12-24). Currently, the mild system is available commercially in the United States and the company is seeking national Medicare reimbursement.

Coverage with Evidence Development (CED) is one option authorized by the Centers for Medicare \& Medicaid Services (CMS) that allows for the collection of clinical data by companies on approved devices to generate evidence to support reimbursement (25). With CED, Medicare covers items and services on the condition that they are provided in the context of approved clinical studies (25). The CED process allows for the collection of clinical data for new and innovative technologies where the treatments are likely to show a benefit for the Medicare population, but where the evidence does not yet provide a "sufficiently persuasive" basis for coverage. The goal is to provide data to assess reimbursement that is well supported by available scientific and medical evidence. CED studies must be in compliance with all applicable Federal regulations concerning the protection of human patients including FDA regulations and the studies or registries must be registered on the www.ClinicalTrials.gov website prior to enrollment of the first study patient. Final results must be reported in a publically accessible manner such as a peer-reviewed journal. MiDAS ENCORE has been approved by CMS.

\section{Study Design}

\section{Objective}

This is a prospective, multi-center, randomized controlled clinical study designed to compare patient outcomes following treatment with either the mild procedure or ESIs in LSS patients exhibiting neurogenic claudication and having verified ligamentum flavum hypertrophy as a contributing factor. The goal of MiDAS ENCORE is to provide Level I evidence of the safety and effectiveness of mild versus ESIs in managing neurogenic claudication symptoms in LSS patients.

\section{Study Sites}

There are 26 interventional pain management centers throughout the United States enrolling patients in MiDAS ENCORE (Table 1). All investigators have been trained on the mild procedure under the same basic training protocol. Further, investigators are required to have clinical experience with both study interventions, having treated a minimum of 10 patients with the mild procedure, as well as with ESI prior to participation in this study.

\section{Study Population}

Approximately 300 Medicare patients who meet the study selection and symptomatic diagnosis criteria will be enrolled in the study. The eligibility of a patient for randomization in this study is determined after all enrollment and symptomatic diagnosis criteria have been satisfied. Inclusion and exclusion criteria are presented in Table 2 . In addition, the following symptomatic diagnosis (26) screening criteria to confirm symptoms of neurogenic claudication will be used prior to patient enrollment in the study:

1. Pain/Discomfort in leg, buttocks, or lower back while walking or standing.

2. Pain relief experienced when bending forward or sitting down. 
Table 1. Enrolling sites and investigators.

\begin{tabular}{|c|c|c|c|}
\hline Investigator's Name & Site & City & State \\
\hline $\begin{array}{l}\text { F. McDonnell, MD (PI) } \\
\text { J. Waling, MD }\end{array}$ & Deaconess Comprehensive Pain Center - West & Evansville & IN \\
\hline $\begin{array}{l}\text { R. Haladjian, MD (PI) } \\
\text { N. Patel, MD }\end{array}$ & Michigan Interventional Pain Center & Brownstown Township & MI \\
\hline W. Von Kaenel, MD (PI) & Spine Intervention Medical Corporation & Fresno & $\mathrm{CA}$ \\
\hline B. Chafin, MD (PI) & Roanoke-Chowan Pain Management & Ahoskie & $\mathrm{NC}$ \\
\hline R. Paicius, MD (PI) & Newport Beach Headache and Pain & Newport Beach & $\mathrm{CA}$ \\
\hline S. Li, MD & Premier Pain Centers & Shrewsbury & NJ \\
\hline $\begin{array}{l}\text { W. Richardson, MD (PI) } \\
\text { M. Netherton, MD }\end{array}$ & Southeastern Spine Institute & Mt Pleasant & SC \\
\hline G. Chartier, MD (PI) & Willow Creek Pain Center & Vincennes & IN \\
\hline S. Golovac, MD (PI) & Florida Pain Institute & Merritt Island & FL \\
\hline S. Kramer, MD (PI) & Kramer Orthopedics & Newport Beach & $\mathrm{CA}$ \\
\hline R. Vallejo, MD (PI) & Millennium Pain Center & Bloomington & IL \\
\hline M. Verdolin, MD (PI) & Synovation Medical Group & Chula Vista & $\mathrm{CA}$ \\
\hline $\begin{array}{l}\text { E. Washabaugh, MD (PI) } \\
\text { L. Bojrab, MD } \\
\text { J. Chatas, MD }\end{array}$ & Michigan Pain Specialists & Ypsilanti & MI \\
\hline $\begin{array}{l}\text { K. Zaffarkhan, MD (PI) } \\
\text { H. Sata, MD }\end{array}$ & Regenerative Institute of Newport Beach & Newport Beach & $\mathrm{CA}$ \\
\hline M. Hanowell, MD (PI) & The Knox Surgical Center & Covington & GA \\
\hline J. Rosenberg, MD (PI) & SC Pain \& Spine Specialists & Murrells Inlet & SC \\
\hline V. Johnson, MD (PI) & The Spine Institute & Murrieta & $\mathrm{CA}$ \\
\hline R. Lingreen, MD (PI) & Frankfort Pain Clinic & Frankfort & KY \\
\hline D. Choi, MD (PI) & Valley Pain Consultants & Scottsdale & $\mathrm{AZ}$ \\
\hline S. Wahezi, MD (PI) & Montefiore Rehab Center & Bronx & NY \\
\hline A. Calodney, MD (PI) & Texas Spine and Joint Hospital & Tyler & $\mathrm{TX}$ \\
\hline E. Frankoski, MD (PI) & Interventional Pain Physicians of South Florida & Aventura & FL \\
\hline $\begin{array}{l}\text { C. Kim, MD (PI) } \\
\text { R. Bowman, MD }\end{array}$ & The Center for Pain Relief & Charleston & WV \\
\hline R. Reinhart, MD (PI) & Desert Pain Management & Rancho Mirage & CA \\
\hline $\begin{array}{l}\text { T. Lamer, MD (PI) } \\
\text { B. Hoelzer, MD }\end{array}$ & Mayo Clinic Pain Management & Rochester & $\mathrm{MN}$ \\
\hline $\begin{array}{l}\text { N. Moghal, MD (PI) } \\
\text { W James, MD }\end{array}$ & Comprehensive Center For Pain Management & Toledo & $\mathrm{OH}$ \\
\hline
\end{tabular}

3. Flexion forward while walking.

4. Inability to stand unaided for more than 15 minutes without bending at the waist.

5. Inability to walk unaided for more than one quar- ter mile without bending at the waist.

6. History of symptoms greater than or equal to 12 weeks. 
Table 2. Inclusion/exclusion criteria.

\begin{tabular}{|l|}
\hline \multicolumn{1}{|c|}{ Inclusion Criteria } \\
\hline 1. 65 years or older and a Medicare beneficiary. \\
2. Patients experiencing neurogenic claudication symptoms for at \\
least 3 months duration who have failed to respond or poorly \\
responded to physical therapy, home exercise programs, and oral \\
analgesics.
\end{tabular}

3. Lumbar spinal stenosis with neurogenic claudication diagnosed via a. Symptomatic diagnosis

and

b. Radiologic evidence of LSS with unilateral or bilateral ligamentum flavum $>2.5 \mathrm{~mm}$ confirmed by pre-op MRI or CT performed within 12 months of baseline visit.

4. Patients with comorbid conditions commonly associated with spinal stenosis, such as osteophytes, facet hypertrophy, minor spondylolisthesis, foraminal stenosis, and/or disc protrusion may be included unless the treating physician has determined that the condition is too advanced.

5. Available to complete 6 month and one year follow-up visits.
1. ODI Score $<31$ ( 0 - 100 ODI Scale).

2. NPRS Score $<5(0-10$ NPRS Scale).

3. Prior surgery at any treatment level.

4. History of spinal fractures with current related pain symptoms.

5. Patients with Grade III or higher spondylolisthesis as defined below*.

* Measuring Grade III spondylolisthesis: Take the sagittal MRI image (T1 or T2) at the midline view and divide each vertebral body endplate into 4 equal sections (quarters). Next, observe any offsets between adjoining vertebral segments (L1-L2, L2-L3, L3L4, L4-L5, L5-S1). If the offset (slippage) is greater than 2 quarters on either adjoining endplate then the patient is deemed to have Grade III spondylolisthesis and would be excluded from the study.

6. Motor deficit or disabling back and/or leg pain from causes other than LSS neurogenic claudication (e.g., acute compression fracture, metabolic neuropathy, or vascular claudication symptoms, etc.).

7. Unable to walk $\geq 10$ feet unaided before being limited by pain. In this context, "unaided" means without the use of a cane, walker, railing, wall, another person, or any other means of walking assistance.

8. Patients previously randomized and/or treated in this clinical study.

9. Patients that have previously received the mild procedure.

10. ESI during 8 weeks prior to study enrollment.

11. Epidural lipomatosis (if it is deemed to be a significant contributor of canal narrowing by the physician).

12. On (or pending) Workman's Compensation or known to be considering litigation associated with back pain.

\section{Randomization}

After satisfying all inclusion/exclusion and symptomatic diagnosis criteria at baseline, patients will be randomized to one of 2 study cohorts (1:1 randomization). The treatment group will undergo decompression using the mild procedure. The control group will undergo ESI treatment. All patients will receive the same preoperative workup and instructions. Randomization will be stratified by study site. A schematic illustration of patient flow will be presented with a flow diagram as recommended by CONSORT $(27,28)$. A chart of the study patient flow is shown in Fig. 1.

\section{Study Interventions}

\section{The mild Procedure:}

The mild procedure is a percutaneous, minimally invasive, lumbar decompression procedure which is performed under contrast-enhanced fluoroscopic guidance through a small 6-gauge port. Mild is performed with only local anesthetic and moderate sedation, and leaves no implants behind. The mild devices are designed to access the interlaminar space from the posterior lumbar spine, enabling removal of small portions of lamina and hypertrophic ligamentum flavum, thereby achieving 


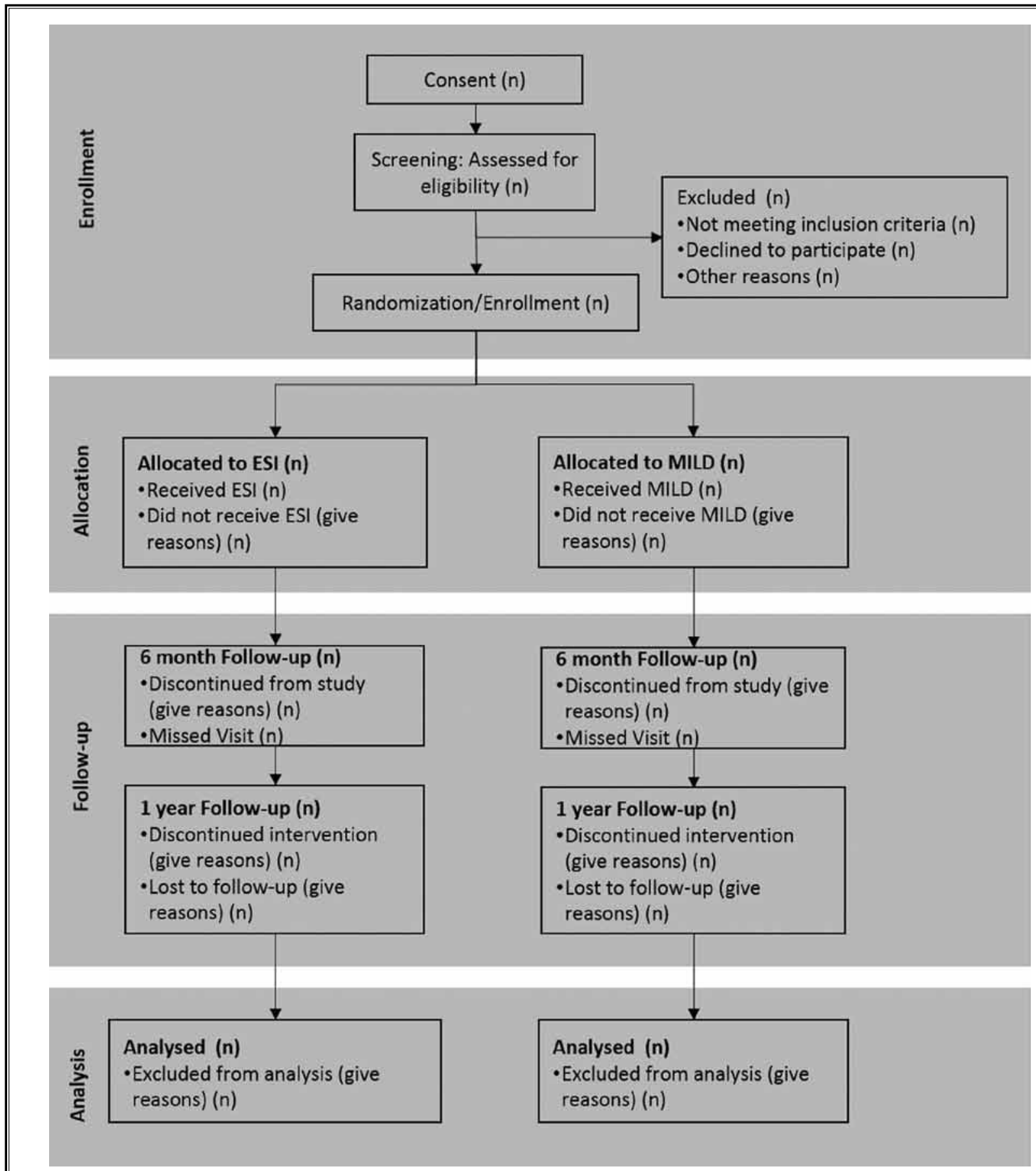

Fig. 1. Presentation of patient flow.

lumbar decompression. Following epidurography, partial decompression is performed through the removal of tissue and bone at the symptomatic level as previously confirmed with correlated magnetic resonance imaging
(MRI) and clinical findings. Epidurography establishes a visual safety margin to avoid penetration of dura. The amount of decompression is assessed through visual observation of epidurogram contrast flow. Pre- and 
post-procedure imaging with contrast (epidurogram) is performed with the patient in the same position and evidence of decompression is confirmed.

\section{Epidural Steroid Injections:}

ESI is performed by appropriately trained physicians utilizing interlaminar treatment at the symptomatic levels. Within the spinal canal, an epidural steroid is injected into the space around the spinal cord and nerve roots (epidural space). During the procedure, fluoroscopic guidance will be used intermittently to guide placement of the needle with the use of contrast. Patients assigned to ESI may have the treatment carried out, according to medical necessity criteria, up to a maximum of 4 times per year in the therapeutic phase consistent with American Society of Interventional Pain Physicians (ASIPP) guidelines (6). The suggested frequency should be 2 months or longer between each injection, provided that $>50 \%$ relief is obtained for 2 months (6). For the initial ESI, $80 \mathrm{mg}$ of kenalog or depo-medrol (40 mg for diabetics) is injected through an epidural needle via a interlaminar approach per standard clinical practice. For subsequent ESI treatments, between 40 and $80 \mathrm{mg}$ can be used.

For either therapy, treatment can be bilateral and at multiple levels. Post-procedure, the patient shall be released at the physician's discretion. Study participants will continue their pre-existing or new conservative management considered appropriate by their treating physician. Co-interventions will be recorded. The use of ESI in the lumbar region for the mild cohort is prohibited during the study period.

\section{Follow-up}

Patients in both treatment arms are required to complete study follow-up evaluations at 6 months and 1 year post-treatment. The total duration of the randomized controlled study is expected to be 24 months, including 12 months for patient recruitment and 12 months for final patient follow-up. Supplementary outcome data will be collected and reported for the mild treatment arm through 1 year.

\section{Outcome Measures and Statistical Methods}

Primary study results will be presented based on one-year follow-up data, with an interim analysis report when 6-month follow-up data are available. Descriptive summaries will be presented by randomized group for all baseline and outcome measures. Inferential statistical analysis will be conducted to determine significant differences related to functional improvement and pain relief, and safety outcomes. Continuous data will be summarized using descriptive statistics: mean, standard deviation, median, minimum, and maximum. Categorical variables will be summarized using frequency counts and percentages. All hypothesis testing will be performed using a 2-sided test at a 0.05 level of significance or a one-sided test at a 0.025 level of significance.

All patients who receive the mild procedure or the traditional ESI therapy will be evaluated for safety and efficacy regardless of whether or not the procedure is aborted or the patient withdraws prior to the completion of all post-treatment evaluations. Prior to the overall analysis, study-site differences will be assessed for all primary and secondary endpoints. If betweensite differences are noted, the appropriate adjustments will be made prior to the overall analysis. Patients will be analyzed on an intent-to-treat (ITT) basis.

\section{Efficacy}

The primary efficacy outcome measure for this study is the proportion of Oswestry Disability Index (ODI) responders from baseline to one year follow-up for both cohorts. ODI (American Academy of Orthopaedic Surgeons-MODEMS [Musculoskeletal Outcomes Data Evaluation and Management Systems] version) is a widely used, validated tool that researchers and disability evaluators use to measure a patient's functional disability (29). ODI ranges from 0 to 100, with lower scores indicating less severe symptoms. ODI responders are defined as those patients achieving the validated Minimal Important Change (MIC) of $\geq 10$ point improvement in ODI score from baseline to follow-up as a clinically significant efficacy threshold $(30,31)$. Patients who do not achieve the clinically relevant efficacy threshold at follow-up, cross over to the alternate therapy arm, receive or intend to receive an invasive non-study procedure in the lumbar region, or voluntarily withdraw due to poor response to the procedure are considered non-responders.

The primary efficacy objective is to demonstrate statistical superiority of mild to ESI on the proportion of ODI responders. The hypothesis will be tested by constructing the 2 -sided $95 \%$ confidence interval around the difference between the population proportions (pmild - pESI). If the lower bound of the 2-sided confidence interval is greater than 0 , superiority will be declared and the endpoint will be met.

Secondary efficacy endpoints include the Zurich Claudication Questionnaire (ZCQ) and the Numeric Pain Rating Scale (NPRS). ZCQ is a validated measure specific 
to LSS. ZCQ is used to assess and quantify severity of symptoms, physical function characteristics, and patient satisfaction after treatment $(15,23)$. According to published validation studies, improvement in ZCQ domains of at least 0.5 indicates a MIC, and a Patient Satisfaction score of at least 2.5 represents a satisfied patient (3235). NPRS records pain on a 0 to 10 scale, from no pain to the worst pain imaginable (36). The MIC difference in NPRS has been established to be 2 points $(30,31,37-$ 39). The analysis of secondary efficacy endpoints will report rates of ZCQ and NPRS responders from baseline to one year follow-up for both cohorts. Supplementary efficacy data will be analyzed and reported for mild patients to 1 year follow-up.

\section{Safety}

The primary safety endpoint is the incidence of device- and/or procedure-related adverse events, which will be compared statistically between the randomized groups using proportions of patients experiencing such an event. The endpoint will be met if this event rate is not significantly greater with mild than with ESI. All patients will be evaluated at baseline, during the treatment procedure, and throughout the follow-up period. All study-related adverse events will be monitored and reported, including seriousness, severity, treatment, and relationship to the study device/procedure. Adverse events collected in this study will be those determined by the site investigators to be specifically related to the products or procedures used in the 2 treatment groups, or one that is determined to be a serious adverse event. All adverse events will be evaluated and adjudicated by the study principal investigators.

\section{Sample Size}

Sample size was calculated to meet the threshold of $80 \%$ power in testing the primary superiority hypothesis. The sample size of 150 patients per group is sufficient under the assumption of a 2-sided hypothesis, type 1 error of 0.05 , power (1- $\beta$ ) at least $80 \%$, randomization ratio of $1: 1$, and accounting for dropouts.

\section{Pain Management}

The investigators may use opioid and non-opioid analgesics as needed by patients in both treatment groups according to the standard of care and if used for leg/back pain, shall be recorded at baseline and follow-up visits. Any changes in a patient's narcotic and/ or non-narcotic medication usage will be documented in real time. In addition, all patients will be provided with a personal patient diary that will enable patients to immediately record dates and details of medication changes throughout the study period.

Other LSS directed back therapies shall be used as needed according to the standard of care (i.e., physical therapy; home exercise programs; walking aids; facet, trigger point, or sacroiliac joint injections, etc.). The use of ESI in the lumbar region for the mild cohort is prohibited during the study period.

\section{Data Collection}

At baseline, patient demographics and prior failed therapies will be documented, including frequency, duration, type, and compliance. Documentation of analgesic use for the treatment of neurogenic claudication symptoms will include frequency, dosage, and duration. All baseline and post-procedural follow-up time points will include reported outcomes documented through completing the ODI, ZCQ, and NPRS questionnaires, and documentation of narcotic and non-narcotic analgesic medication usage specific to neurogenic claudication and co-interventions.

All patients will be evaluated at baseline, during the treatment procedure, and at 6 months and one year post-treatment. Device and procedure-related adverse events, and all serious adverse events will be documented throughout the study period (Table 3.)

\section{Study Governance}

The study principal investigators are responsible for study oversight and reporting of results. Responsibilities of the study principal investigators include protocol review, assistance with site selection, site investigator support, oversight of patient enrollment and protocol compliance, and adjudication of adverse events. The study principal investigators will not be involved in treating patients, are blinded to study outcome data, and will receive only 6-month and one-year summary clinical reports prepared by the independent statistician. The sponsor is also blinded to study outcome data. All sites must obtain Institutional Review Board approval and patients must provide written informed consent in accordance with the local clinical site's IRB. Patient confidentiality will be maintained under HIPAA Privacy Rules and Good Clinical Practice guidelines for clinical research will be followed. The study is designed to meet the regulatory requirements of the Food and Drug Administration responsibilities as applicable including sponsor general duties (21 CFR Part 812.40), selection of investigators (21 CFR Part 812.43), monitor- 
Table 3. Schedule of assessments.

\begin{tabular}{|l|c|c|c|c|c|}
\hline \multicolumn{1}{|c|}{ Assessments } & $\begin{array}{c}\text { Screening } \\
\text { I Baseline }\end{array}$ & Procedure & 6 Months & One Year & $\begin{array}{c}\text { Supplementary Data- } \\
\text { mild Patients Only } \\
\text { at 2 Years }\end{array}$ \\
\hline Patient Informed Consent & $\mathrm{X}$ & - & - & - & - \\
\hline Selection and Symptomatic Diagnosis Criteria & $\mathrm{X}$ & - & - & - & - \\
\hline Lumbar Spine History & $\mathrm{X}$ & - & - & - & - \\
\hline Narcotic and Non-Narcotic Analgesic Usage Collection & $\mathrm{X}$ & $\mathrm{X}$ & $\mathrm{X}$ & $\mathrm{X}$ & $\mathrm{X}$ \\
\hline Conservative Therapy / Co-Interventions & $\mathrm{X}$ & - & $\mathrm{X}$ & $\mathrm{X}$ & $\mathrm{X}$ \\
\hline Oswestry Disability Index (ODI) & $\mathrm{X}$ & - & $\mathrm{X}$ & $\mathrm{X}$ & $\mathrm{X}$ \\
\hline Zurich Claudication Questionnaire (ZCQ) & $\mathrm{X}$ & - & $\mathrm{X}$ & $\mathrm{X}$ & $\mathrm{X}$ \\
\hline Numeric Pain Rating Scale (NPRS) & $\mathrm{X}$ & - & $\mathrm{X}$ & $\mathrm{X}$ & $\mathrm{X}$ \\
\hline MRI Record of Spine L1-S1 & $\mathrm{X}$ & - & - & - & - \\
\hline Study Procedure / Treatment Information & - & $\mathrm{X}$ & - & - & $\mathrm{X}$ \\
\hline Significant Adverse Events & - & $\mathrm{X}$ & $\mathrm{X}$ & $\mathrm{X}$ & $\mathrm{X}$ \\
\hline Follow-Up Visits & - & - & $\mathrm{X}$ & $\mathrm{X}$ & $\mathrm{X}$ \\
\hline
\end{tabular}

Visit window: 6 months \pm 14 days; 1 year \pm 30 days; 2 years \pm 45 days

ing (21 CFR Part 812.46) and maintaining records (21 CFR Part 812.140 (b)). This study has been registered in the U.S. Clinical Trial Registry (NCT02093520).

\section{Discussion}

Use of ESIs for the treatment of low back pain has been broadly accepted, and ESIs are generally recognized as the standard of care after failure of conservative therapy (3-11). It is important to note, however, that low back pain, and specifically LSS, can be caused by different pathophysiological mechanisms leading to pain and reduced mobility, including radicular pain and neurogenic claudication. Radicular pain is related to inflammation of the spinal nerve roots, and is expressed in a dermatomal pain pattern. Conversely, neurogenic claudication symptoms are caused by ischemia resulting from compression of the nerve roots. Many patients suffer from both radicular pain and neurogenic claudication, however this study is focused specifically on the treatment of LSS patients suffering from neurogenic claudication with ligamentum flavum hypertrophy as a contributing factor to central canal stenosis.

There is one prior published study that directly compared the use of ESI and mild for the treatment of LSS patients with painful lower limb neurogenic claudication and hypertrophic ligamentum flavum as a contributing factor (13). The study was a double-blind, randomized prospective study conducted at a single pain management center. In order to maintain study patient blinding, only one ESI treatment was administered via an interlaminar approach for those patients randomized to the ESI cohort. In that study, the results demonstrated that in LSS patients, mild provided statistically significantly better pain reduction and improved function than those treated with ESI at 6 weeks.

Lack of patient blinding is a limitation of this study. Due to significant differences in treatment protocols between the 2 study groups, including the use of multiple ESI treatments throughout the study period, patient blinding is not feasible. It should be noted however that all patients are instructed to complete the patient reported outcome questionnaires on their own, and are provided a quiet setting.

Another potential limitation of the study is that neither study arm is focused on the treatment of radicular pain. In fact, there are restrictions in the study protocol that limit additional and adjunctive pain treatments, many of which would be standard of care in normal practice. As a result, there is no expectation that patients will be pain-free. These restrictions are required to allow for a direct comparison of the 2 treatment arms in this randomized controlled study. One potential negative impact of these restrictions is that there may be a higher non-responder rate for both groups within the study versus standard of care outside the confines of a clinical study.

\section{Conclusion}

This prospective, multi-center, randomized controlled study will provide Level I evidence of the safety and effectiveness of mild versus ESIs in managing neurogenic claudication symptoms in LSS patients. 


\section{Acknowledgments}

The authors wish to thank Debbie Barber, MS, an independent contractor, for her assistance in prepara- tion of this manuscript. The authors would also like to thank the editorial board of Pain Physician for review and criticism in improving the manuscript.

\section{References}

1. Porter RW. Spinal stenosis and neurogenic claudication. Spine 1996; 21:2046-2052.

2. Simotas AC, Dorey FJ, Hansraj KK, Cammisa F. Nonoperative treatment for lumbar spine stenosis-Clinical and outcome results and a 3-year survivorship analysis. Spine 2000; 25:197-203.

3. Manchikanti L, Cash KA, McManus CD, Pampati V, Fellows B. Results of 2-year follow-up of a randomized, doubleblind, controlled trial of fluoroscopic caudal epidural injections in central spinal stenosis. Pain Physician 2012; 15: 371-384.

4. Manchikanti L, Cash KA, McManus CD, Damron KS, Pampati V, Falco FJE. A randomized, double-blind controlled tria of lumbar interlaminar epidural injections in central spinal stenosis: 2-year follow-up. Pain Physician 2015; 18:79-92.

5. Manchikanti L, Kaye AD, Manchikant KN, Boswell MV, Pampati V, Hirsch JA. Efficacy of epidural injections in the treatment of lumbar central spinal stenosis: A systematic review. Anesth Pain Med 2015; 5:e23139.

6. Manchikanti L, Abdi S, Atluri S, Benyamin RM, Boswell MV, Buenaventura RM, Bryce DA, Burks PA, Caraway DL, Calodney AK, Cash KA, Christo PJ, Cohen SP, Colson J, Conn A, Cordner H, Coubarous S, Datta S, Deer TR, Diwan S, Falco FJ, Fellows B, Geffert S, Grider JS, Gupta S, Hameed H, Hameed $M$, Hansen $\mathrm{H}$, Helm $\mathrm{S}$ 2nd, Janata JW, Justiz R, Kaye AD, Lee M, Manchikanti KN, McManus CD, Onyewu O, Parr AT, Patel VB, Racz GB, Sehgal N, Sharma ML, Simopoulos TT, Singh V, Smith HS, Snook LT, Swicegood JR, Vallejo R, Ward SP, Wargo BW, Zhu J, Hirsch JA. An update of comprehensive evidence-based guidelines for interventional techniques in chronic spinal pain. Part II: Guidance and recommendations. Pain Physician 2013; 16:S49-S283.

7. Manchikanti L, Pampati V, Falco FJE, Hirsch JA. An updated assessment of utilization of interventional pain man- agement techniques in the Medicare population: $2000-2013$. Pain Physician 2015; 18:E115-E127.

8. Friedly JL, Comstock BA, Turner JA, Heagerty PJ, Deyo RA, Sullivan SD, Bauer Z, Bresnahan BW, Avins AL, Nedeljkovic SS, Nerenz DR, Standaert C, Kessler L, Akuthota V, Annaswamy T, Chen A, Diehn F, Firtch W, Gerges FJ, Gilligan C, Goldberg $H$, Kennedy DJ, Mandel S, Tyburski M, Sanders W, Sibell D, Smuck M, Wasan A, Won L, Jarvik JG. A randomized trial of epidural glucocorticoid injections for spinal stenosis. N Engl J Med 2014; 371:11-21.

9. Manchikanti L, Candido KD, Kaye $A D$, Boswell MV, Benyamin RM, Falco FJE, Gharibo CG, Hirsch JA. Randomized trial of epidural injections for spinal stenosis published in the New England Journal of Medicine: Further confusion without clarification. Pain Physician 2014; 17:E475-E488.

10. Liu K, Liu P, Liu R, Wu X, Cai M. Steroid for epidural injection in spinal stenosis: A systematic review and meta-analysis. Drug Des Devel Ther 2015; 9:707-716.

11. Manchikanti L, Cash KA, McManus CD Pampati V. Assessment of effectiveness of percutaneous adhesiolysis in managing chronic low back pain secondary to lumbar central spinal canal stenosis. Int J Med Sci 2013; 10:50-59.

12. Basu S. Mild procedure single-site prospective IRB study. Clin J Pain 2012; 28:254-258.

13. Brown L. A double-blind, randomized, prospective study of epidural steroid injection vs. the mild® procedure in patients with symptomatic lumbar spinal stenosis. Pain Pract 2012; 12:333-341.

14. Chen $\mathrm{H}$, Kelling J. mild procedure for lumbar decompression: A review. Pain Pract 2013; 13:146-153.

15. Chopko BW, Caraway D. MiDAS I (mild decompression alternative to open surgery): A preliminary report of a prospective, multi-center clinical study. Pain Physician 2010; 13:369-378.
16. Chopko BW. Long-term results of percutaneous lumbar decompression for LSS: Two-year outcomes. Clin J Pain 2013; 29:939-943.

17. Deer T, Kapural L. New image-guided ultra-minimally invasive lumbar decompression method: The mild ${ }^{\otimes}$ procedure. Pain Physician 2010; 13:35-41.

18. Deer T, Kim C, Bowman II R, Ranson M, Yee BS. Study of percutaneous lumbar decompression and treatment algorithm for patients suffering from neurogenic claudication. Pain Physician 2012; 15:451-460.

19. Deer T, Mekhail N, Lopez G, Amirdelfan A. Minimally invasive lumbar decompression for spinal stenosis. JNR 2011; 1:29-32.

20. Lawrence MM, Hayek SM. Minimally invasive lumbar decompressions: A treatment for lumbar spinal stenosis. Curr Opin Anaesthesiol 2013; 26:573-679.

21. Levy R, Deer T. Systematic safety review and meta-analysis of procedural experience using percutaneous access to treat symptomatic lumbar spinal stenosis. Pain Med 2012; 13:1554-1561.

22. Mekhail N, Costandi S, Abraham B, Samuel, SW. Functional and patient-reported outcomes in symptomatic lumbar spinal stenosis following percutaneous decompression. Pain Pract 2012; 12:417-425.

23. Mekhail N, Vallejo R, Coleman $\mathrm{MH}, \mathrm{Be}-$ nyamin RM. Long-Term results of percutaneous lumbar decompression mild for spinal stenosis. Pain Pract 2012; 12:184-193.

24. Schomer DF, Solsberg D, Wong W, Chopko BW. mild® Lumbar decompression for the treatment of lumbar spinal stenosis. Neuroradiology J 2011; 24:620-626.

25. Guidance for the Public, Industry, and CMS Staff Coverage with Evidence Development Document Issued on November 20, 2014: www.cms.gov/ medicare-coverage-database/details/ medicare-coverage-document-details. 
aspx?MCDId=27.

26. Sandella DE, Haig AJ, Tomkins-Lane C, Yamakawa K. Defining the clinical syndrome of lumbar spinal stenosis: A recursive specialist survey process. PMR 2013; 5:491-495.

27. Moher D, Schulz KF, Altman D; CONSORT Group. The CONSORT statement: Revised recommendations for improving the quality of reports of parallelgroup randomized trials. JAMA 2001; 285:1987-1991.

28. Piaggio G, Elbourne DR, Altman DG, Pocock SJ, Evans S); CONSORT Group. Reporting of noninferiority and equivalence randomized trials: An extension of the CONSORT statement. JAMA 2006; 295:1152-1160.

29. Fairbank NCT, Pynsent PB. The Oswestry Disability Index. Spine 2000; 25:2940-2952.

30. Hägg O, Fritzell P, Nordwall A; Swedish Lumbar Spine Study Group. The clinical importance of changes in outcome scores after treatment for chronic low back pain. Eur Spine J 2003; 12:12-20.
31. Ostelo RWJG, Deyo RA, Stratford P, Waddell G, Croft P, Von Korff M, Bouter LM, de Vet HC. Interpreting change scores for pain and functional status in low back pain towards international consensus regarding minimal important change. Spine 2008; 33:90-94.

32. Stucki G, Liang MH, Fossel AH, Katz JN. Relative responsiveness of conditionspecific and generic health status measures in degenerative lumbar spinal stenosis. J Clin Epidemiol 1995; 48:1369-1378.

33. Stucki G, Daltroy L, Liang MH, Lipson SJ, Fossel AH, Katz JN. Measurement properties of a self-administered outcome measure in lumbar spinal stenosis. Spine 1996; 21:796-803.

34. Zucherman JF, Hsu KY, Hartjen CA, Mehalic TF, Implicito DA, Martin MJ, Johnson DR 2nd, Skidmore GA, Vessa PP, Dwyer JW, Puccio S, Cauthen JC, Ozuna RM. A prospective randomized multi-center study for the treatment of lumbar spinal stenosis with the X STOP interspinous implant: 1-year results. Eur Spine J 2004; 13:22-31.
35. Moojen WA, Arts MP, Brand R, Koes BW, Peul WC. The Felix-trial. Double-blind randomization of interspinous implant or bony decompression for treatment of spinal stenosis related intermittent neurogenic claudication. BMC Musculoskel Disord 2010; 11:100.

36. Williamson A, Hoggart B. Pain: A review of three commonly used pain rating scales. J Clin Nurs 2005; 14:798-804.

37. Childs JD, Piva SR, Fritz JM. Responsiveness of the numeric pain rating scale in patients with low back pain. Spine 2005; 30:1331-1334.

38. Farrar JT, Young JP Jr, LaMoreaux L, Werth JL, Poole RM. Clinical importance of changes in chronic pain intensity measured on an 11-point numerical pain rating scale. Pain 2001; 94:149-158.

39. Salaffi $F$, Stancati A, Silverstri CA, Ciapetti A, Grassi W. Minimal clinically important changes in chronic musculoskeletal pain intensity measured on a numerical rating scale. Eur J Pain 2004; 8:283-291. 